\title{
Le Troisième Congrès de la Houille Blanche
}

\author{
Organisé par la Chambre Syndicale des Forces Hydrauliques, de l'Electrochimie, de l'Electrométallurgie \\ et des Industries qui s'y rattachent
}

\section{A GRENOBLE, des $4^{\prime}$ au 8 Juillet 1925}

par Victor SyLvestre, Ingénicur A. M. et I. E. G.,

Secrélaire de la Classe VI "Gros matériel électrique " de l'Exposilion Internationale de la Houille Blanche et du Tourisme.

"Ainsi une grande révolution esl accomplie; la monlagne, jusque-là inactive el inféconde, va prendre " part au labeur universel; elle est domptée et mâttrisée par son propre fils : le montagnard.

Gabricl Havotaux,

(Congrès de la Houille Blanche, 1902).

"Si Bergìs, Fredet et Matussière ont laissé des héritiers qui portent dignement leur nom, ils en ont laissé " une infinite d'autres qui sont leur fils spiriluels. Nous sommes, nous aussi, les pionniers de la Houille "Blanche, nous avons l'orgueil de continuer l'ur chuvre, et notre seule ambition est de poursuivre sans arrêt " le sillon creusś par ces hons oubriers de la première heure.

Louis Marlio,

(Discours du Plateau des Petites Roches, 5 juillet 1925. Troisième Congrès de la Honille Blanche.

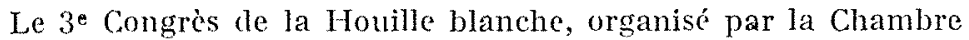
symdicale des Forces hydrauliques, de l'Electrochimie, de l'Electromctallurgie et des industries s'y rattachant, s'est ouvert à Grenoble le samedi 4 juillet 1925 , à neuf heures, en séance inaugurale, à l'amphithéatre du nouvel Institut Polytechnique, rue Général- blanche. Les invitations furent lancées; mais, malheureuscment, la terrible guerre vint arrèter toutes les initiatives.

M. Marlio, en ouvrant, le 4 juillet 1925 , le $3^{e}$ Congrès de la Houille blanche, adressa ses premières paroles de gratitude à tous ceux qui apportèrent leur concours : au président de la République, M. Dou-

\section{LES PRECURSEURS DE LA " HOUILLE BLANCHE"}

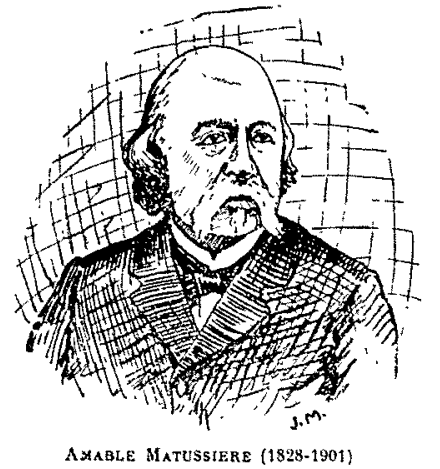

Motte, sous le haut patronage de M. Doumergue, président de la République, et sous la présidence de $M$. Louis Marlio, président de la Chambre syndicale des Forces hydrauliques, de MM. Barut, Bouchayer, Gall, Pinot, et sous la direction de MM. Bachellery, Bougault, de la Brosse, Eydoux, Flusin, Mahieu, Margot, Maroger, Rateau, Fredel, Keller, Lépine, Lacroix, Perinel, Rochette, Tochon Meynial, ctc.

Ce Congrès, qui avait lieu pour la $2^{\circ}$ fois à Grenoble, groupait plus de 600 savants et techniciens de France et du monde entier.

M. Louis Marlio, président du Congrès, dans une brillante allocution, a rappelé tout d'abord qu'il y avait 23 ans que la ville de Grenoble, sous les auspices de la Chambre syndicale des Forces hydrauliques, inaugurait le premier congrès de la Houille blanche, en 1902, sous la présidence de M. Charles Pinat, de M. Cordier et de M. Gabriel Hanotaux, de l'Académie française. Le mot « houille blanche n, dû à Aristicle Bergès, allait faire fortune ; les techniciens et les industricls se pressaient nombreux pour faire fète à l'industrie naissante et afin de nettre de l'ordre dans tous les problèmes nouveaux qui se posaient à eux. De nombreux touristes se joignaient aux différents groupements techniques. Douze ans plus tard, au printemps 1914 , était convoqué le $2^{\circ}$ Congres de la Houille mergue; aux délégués du Gouvernement et en particulier à M. Cordier, et aux trois présidents des sections : M. Wahieu, pour la section économique: $M$. Rateau, menbre de l'Institut, pour la section technique; M. Eydoux, son adjoint; à M. de la Brosse, pour la section administrative; aux différents ingénieurs, industriels, financiers, juristes, qui ont rérligé des rapports remarquables qui feront date et qui furent discutés dans les différentes commissions qui tinrent leurs assises à la Chambre de commerce; au Comité consultatif des Forces hydrauliques: aux conseillers d'Etat; aux représentants étrangers qui ont bien voulu honorer ce Congrès de leur compétence.

M. Marlio a fait ressortir que ce qui avail motivé le $3^{e}$ Congrès de la Houille blanche était l'Exposition internationale de Grenoble, et il a remercié en termes chaleureux $M$. Marius Blanchet, commissaire général, M. Flusin, M. Keller et M. Lépine.

Il a rappelé également que d'autres motifs appelaient un troisième Congrès de la Honille blanche : d'abord l'énorme croissance de l'industrie derla houille blanche depuis ses débuts, qui datent de 1869 en Dauphiné, croissance représentée par les chiffres suivants : 200.000 chevaux installés en puissances hydrauliques en 1902; 750.000 en $1914 ; 2.000 .000$ en 1924 , et certainement 2.500 .000 che- 
vaux installés en puissances hydrunliques en 1925, el comme capitaux investis de deux cents a huit cents millions entre 1902 et 1911 , et de huit cents à plus de deux milliarels entre 191.1 et $1925: 1^{\circ}$ application de la législation nouvelle résultant de la loi de 1919 et de la dévalorisation du franc résultant de la crise financière que nous traversons, qui a une grande répereussion dans le prix de l'énergie électrique.

Il a fait justement ressortir que les meilleures chutes sont deja amenagées et que le restant de notre domaine by dro-électrique ne poumra ìtre aménagé gu 'i un prix relativenent ćlevé. Il a fait un appel pressant aux financiers, aux technieiens et aux hauts fonctionmares presents au Congres, pour qu'ils se metlent d'accord et qu'ils compremont que. lorsqu'il s'agit d'ume grande cause, comme colle de l'aménagement hydro-électrique en France, il ne doit y avoir qu'un seul point de vue : le point de vue national.

Il a fait ressortir. d'une facon frappante, cu'il n'a jamais été plus nécessaire qu ’à lheure actuclle de développer notre industric de la houille blanche par rapport à la houille noire, afin d'éviter au pays les sorties d'argent nécessaires pour acheter à l'étranger la houlle dont nous avons besoin, pour permettre d'effectuer, d'une façon économique, l'électrification de nos campagnes, de nos chemins de fer, etc.

Il a montré combien le problème était vaste, et il a déclaré ouvert le $3^{\mathrm{e}}$ Congrès de la Houille blanche et souhaite bonne réussite a ce Congrès.

M. Tochon, secrétaire général du Congrès, a pris ensuite la parole, afin de donner des précisions sur l'organisation des différentes sćances de travail, tes conférences et des visites d'usines.

Puis le délégué du gouvernement italien, dans une chaleureuse allocution, a remercié le président du Congrès d'avoir bien voulu l'inviter à cette manifestation; il a rappelé que l'illustre ministre piémontais, Cavour, dans un discours qu'il prononça au moment de l'inauguration du tumel du Mont-Cenis, s'était déjà servi du mot de "Houille blanche", et il a été très applaudi lorsqu'il a dit qu'en toutes occasions, aussi bien dans les périodes de paix que dans les périodes troublées, les techniciens italiens marcheraient la main dans la main avec les techniciens français.

Les différents techniciens se rendirent ensuite à la Chambre de commerce, où les diverses sections commencèrent leurs travaux : la section technique sous la présidence de $\mathrm{M}$. Rateau, membre de l'Institut; de M. Margot, directeur de la Compagnie P.-L.-M. ; de M. Auguste Blanchet, de la Société Hydro-technique de France; de M. Eydoux, professeur à l'Ecole nationale des Ponts et Chaussées; la section administrative, sous la présidence de M. de La Brosse, inspecteur général des Ponts et Chaussées; de M. Aimé Bouchaver, vice-président de la Chambre de commerce de Grenoble, et la section économique et financière. sous la présidence de M. Mahieu, inspecteur des Ponts et Chaussées; de M. Celier, conseiller d'Etat, et de M. Wilratte, directeur de la Banque de Paris et des Pays-Bas.

\section{Lr Banguet du Plateau des Petites Rocins du 5 jullet}

La Chambre syndicale des Forces hydrauliques a organisó, le dimanche 5 juillet, à l'occasion du 3 e Congrìs de la Houille blanche une grande fête au plateau des Petites Roches, en l'honneur et à la gloire des trois précurseurs de la Houille blanche : Bergès, Fredet et Matussière.

A midi, tout à côté de la station du funiculaire, sur la terrasse d'où l'on surplombe la pittoresque vallée du Grésivaudan et l'imposant massil de Belledonne, plus de 300 convives s'installaient sous une immense tente coquettement décorée et payoisée.

Autour de M. Louis Marlio, président de la Chambre syndicale des Forces hydrauliques, avaient pris place Mme Henri Fredet, Mme Matussière; MM. Léon Perrier, sénateur, président du Conseil général; Déléon, des usines Bergès; Marius Blanchet, commissaire général de l'Exposition; Mme Gabriel Forest ; Mme Pierre Matussière; MM. Bourrat. secrétaire général de la préfecture; Albert Mahicu, sénateur, ancien ministre; Mme la comtesse de Foresta; Mme del Buono; M. Henri Fredet, industriel ì Brignoud.

Aux autres tables d'honneur, noté MM. Flusin, commissaire général adjoint de l'Exposition; Lépine, président de la Chambre de commerce; Aimé Bouchayer, président de l'A.P.A.F. et du Conité local d'organisation; de La Brosse el Armand, inspecteurs généraux des Ponts et Chaussées; Ch.-A. Keller: Jules Barrut, Henry Gall et Robert Pinot, vice-présidents de la Chambre syndi- cale des Fores hydraulic|ues; del 13uono, ingénieur a Rome; Forli Angelo, ingénieur à Milan: Henri Cahen, président du Syndicat professionnel des l'roducteurs el Distribuleurs d'électricilé; Gatu, doyen de la Faculté des sciences; le colonel Grapho, du sous-secrétariat de l'Aéronautique; Barbillion, directeur de l'Institut polytechnique: Houpeurt. ingénieur en chef ; le commandant Lazard, du Comité consultatif des Forces hyclraulicues ; Waltier, directeur des Voies navigables ef des Ports maritimes; Hippolyte Bouchayer, Pierre Matussiere, Pierre Fredet, Joya, Henry Forest, inclustriels; Aubry, vice-président honoraire de la Chambre syndicale des Forees hydrauliques: Vennin, directeur général de la Société générale d'entreprises; 'Thury, ingénieur a Genéve: 'Tur, inspecteur général des Ponts et Chaussées; de Seynes, administrateur de la Chambre syndicale des Forees hydrauliques; Hugo Stunz, ingénieur, délégué de la République Argentine; Sacchi Lodisyrolo, inspecteur gencral i la direction des Travaux hydrauliques d'Italie; Tissot, ingénieur a baile: le comte de Foresta: Wyssling, professeur a l'boole polytechnique fédérale de Zurich; Maroger, président de l'Union des producteurs des Pyrénées-Orientales; Johanslen, ingénieur à Oslo (Norvège), Gruner, ingénieur à Bâle: Menjou et Gros, administrateurs de la Chambre syndicale des Forces hydrauliques: Bruchi, ingénieur à Zurich; Sylvestre, vice-prósident de la "Houille blanche".

L'ordomancement était parfait et l'on peut dire que M. ITenri Fredet. Faimable animateur du plateat des Petites-IRoches, et H. G. Tochon, le si actif et si complaisant secrétaire général du Congrès, ont déployé, en la circonstance, toutes les séductions du parfait amphytrion.

Discours de M. Robert PINot, secrélaire général du Comilé des Forg's.

Au dessert, M. Robert Pinot souhaita une cordiale bienvenue aux congressistes, au nom de l'Association métallurgicue el minière contre la tuberculose et du Comité cles Forges, qui sont devenus les principaux propriétaires du plateau.

Il félicite plus particulièrement les techniciens de la Houille blanche, qui, en ce jour de recueillement, sont venus en ce lieu apporter leur souvenir aux ancêtres de cette industrie, qui pril naissance au pied mème de ce plateau des Petites-Roches.

M. Pinot rappelle ce que l'Association métallurgique et minière a entrepris de réaliser sur ce plateau pour lutter contre le pire des fléaux sociaux et tendre une main secourable aux collaborateurs ouvriers frappés par la maladie.

Discours de M. Marlio, président de la Chambre syndicale des Forces hydrauliyues et du troisième Congris de la Houlle blanche.

M. Marlio, président de la Chambre syndicale des Forces hydrauliques, se fait ensuite, avec une rare éloquence, l'interprète des sentiments des congressistes :

\section{Mesdames,}

Messieurs,

Nous nous sommes réunis aujourd'hui pour fèter la mémoire de ceux qui furent les précurseurs et les apôtres de la Houille nlabche, j'ai nommé Bergès et Matussière, et c'est pourquoi mon premier geste sera de m'incliner respectueusement clevant leurs descendants et de dire, à tous ceux qui ont bien voulu accepter notre invitation, ou qui se sont fait représenter, à la fois notre admiration pour le nom dont ils ont hérité et pour la façon clont ils savent le porter.

Pourquoi, me demanderez-vous, avoir choisi pour cette cérémonie filiale, cet endroit écarté, ce plateau solitaire? La réponse sera simple. Pour une fête de la Houille blanche, pouvions-nous choisir un emplacement mieux approprié que le Plateau des PetitesRoches, d'où nous pouvons arlmirer ces immenses glaciers qui constituent des réserves inépuisables d'énergie hydraulique? Pouvions-nous sothaiter un meilleur observatoire, pour contempler d'un seul coup d'cil les emplacements choisis par nos précurseurs pour y équiper les premières chutes de haute montagne, e'est-à-dire Lancey et Brignoud. C'est ce gui donne à notre excursion dominicale le caractère d'un véritable pélerinage au berceau de la Houille blanche.

Enfin, il nous plaît que cette fête, qui rappelle la naissance d'une grande industrie française et mondiale, que cette fête qui altire ici même tant de chef's d'entreprises, tant de directeurs d'usines; 
tant d'ingénicurs, se déroule sur ce plateau où commencent à s'élever ces grandls sanatoria, que la générosité d'une grande industrie française a cutrepris pour défendre la santé de ses ouvriers contre les ravages de la tuberculose. Il y a là comme un symbole at nous voulons que cetle fête, qui est celle de trois grands patrons, soit aussi celle de tous leurs collaborateurs, employés et ouvriers, montrant ainsi qu'entre le travail des uns el le travail des autres, il n'y a pas de fossé que l'on ne puisse franchir, mais une association où chacun apporte le maximum d'effort, pour obtenir le meilleur résultat.

M. Marlio, en une envolée d'une poésie impressionnante, nous dit ensuite comment la vic matérielle de l'homme, aussi bien que la vie économique et poli-

tique des nations, se trourvent guidées, conditionnées, dominées par l'utilisation de l'cau sous toutes ses formes.

Après avoir rendu surtout hommage à Fournesron, qui après avoir inventé In turbine qui porte son nom, créa dans la Forêt Noire vers 1830 , une premiere chute de moyenne hauteur, M. Marlio constate que le progrès s'arrêle. Trente ans se passent. Puis, brusquement, trois hommes s'attaquent au même probleme. En trols ans, la vallée du Grésivaưdan voit surgir les premières chutes de haute montagne. Désormais, l'exemple donné ne sera plus perdu et le progrès ne s'arrètera plus.

L'une des caractéristi(fues de la découverte de la Houille blanche, e'est qu'elle est liée d'une façon intime à l'industrie du papier qui a fait longtemps et qui fait encore la fortune du Dauphiné.

C'est Matussière, le premier qui, abandonnant le papier de chiffon, concoit le projet d'installer à Domène l'industrie de la pâte de bois cui nécessite une grande quantité de force dans une région qui n'a que peu de charbon. Il a l'idée que la Houille blanche peut

transformer cette industrie. Il se passionne pour cette conception ; il lui apporte toute sa puissance de caractère, sa volonté de fer, son activité intrépide. Comme il n'est pas technicien, il soumet son plan à ses amis, il les pousse et les soutient associé ou non avec eux et il est le véritable animateur de ceux qui seront bientôt des maîtres et qui s'appellent Bergès, Fredet. Ceux-ci ont reç la bonne parole, qui ont eu confiance dans l'avenir de la Houille blanche, reprennent les projets primitifs, les transforment, les amplifient, et c'est ainsi qu'en peu de mois, à Lancey et à Brignoud, on voit surgir les premières grandes chutes de montagne de 200 et de 160 mètres, qui ont illustré les noms de Bergès et de Fredet.

Les recherches du savant sont orientées par les besoins de la réalité qui, à chaque époque, met au premier plan un problème déterminé. D'autre part, l'œuvre du savant, aussi bien que les aspirations de l'opinion publicque, sont dominées tous les deux par les progrès de la technique et les possibilités de réalisation. Il arrive donc un moment où l'invention est mûre, où chacun sent qu'eile va surgir comme un beau fruit. Mais, le mérite de l'inventeur demeure assez grand, puisque c'est lui qui, à un moment domné, jette les ponts entre le rêve et la réalité. C'est pourquoi je me plais à voir dans la cérémonie où nous évoquons les noms de Bergès, Fredet, Matussière, non pas l'œuvre de trois hommes isolés, mais l'œuvre de trois grands cerveaux quí sc sont attachés à un même problème avec toute leur énergie, et même l'œuvre de tout un groupe d'hommes qui ont collaboré avec eux, qui ont discuté avec eux les dimensions des machines, la forme des turbines et qui ont apporté dans leur réalisation tout leur art et toute leur amitié. C'est parce qu'ils avaient confiance' dans le résultat final que tous ces collaborateurs qu'il serait injuste d'oublier, fournisseurs, constructeurs, banquiers, se serrèrent antour d'eux pour leur permettre de traverser les heures difficiles, et c'est pourquoi cette journée prend, à mon sens, un caractère symbolique et que je la vois comme la glorification non pas seulement de trois hommes, mais non pas seulement d'une région, de toute une industrie, qui de Grenoble, a débordé sur le monde entier. Peut-on oublier que ces hommes, qui créaient dans le Dauphiné deux industries nouvelles, celle du papier et celle de la houille blanche, ne s'estimaient jamais satisfaits par l'cuvre réaliséc et qu'ils voyaient toujours mieux, toujours plus loin et toujours plus grand? Peut-on oublier que c'est encore Bergès qui équipait le prenier une chute de 500 mètres, qui percait le premier un lac réservoir à 25 mètres de profondeur et qui, dans son apostolat désintéressé, dressait le premier l'inventaire des chutes d'eau françaises ?

C'est que ces trois précurseurs que nous admirons tous étaient, non seulement des industriels ou des ingénieurs, mais des hommes de srand caractère et de grande énergie ayant confiance en euxjicmes et fol dans l'avenir. C'est qu'ils étaient guidés par un idéal auquel ils sacrifiaient tout.

Leur exemple n'it pas été perdu. I.eurs héritiess directs ont conservé intact et développé le patrimoine d'honneur qu'jls avaient recu d'eux. Peuton oublier la place émjnente que les descendants de Matussière ont tenu dans l'industrie de la papeterie francaise et le degré de perfection qu'ils ont donné à leur ancienne usine de Domène comme à leur grande usine de Modane ?

Peut-on oublier que les fils d'Aristide Bergès ont prouvé que la meilleure facon d'honorer leur père était de continuer son cuvre en équipant en France, en Italie, en Espagne, de superbes domaines hydrauliques ? Peut-on oublier enfin qu'Henri Fredet a puisé dans l'héritage paternel ces qualités de sérieux et de hardiesse qui ont marqué son œuvre industrielle et. puisque l'occasion s'en présente, j'aurai plaisir à lui redire aujourd'hui la gratitude que nous lui devons pour la belle ceuvre de la régularisation de la Romanche dont il est véritablement le créateur et qui vient, grâce à l'appui de nos amis du ministère dés Travaux publies et à l'intervention persomnelle de $M$. le sénateur Perrier, de recevoir la sanction définitive du Parlement.

Si Bergès, Fredet et Matussière ont laissé des héritiers qui portent dignement leur nom, ils en ont laissé une infinité d'autres qui sont leurs fils spirituels. Nous sommes, nous aussi, des pionniers de la Houille blanche, nous avons l'orgueil de continuer leur cuvre et, notre seule ambition est de poursuivre sans arrêt le sillon ureusé par ces bons ouvriers de la premicre heure (Vifs upplaudissements). 


\section{Discours de M. Hemri Fredet.}

\section{Mon cher Président,}

.Te dois à mes fonctions d'alministrateur de la Chambre syndicale des Forces hydraulicyues et à la bonne grice des descendants de MU. Bergès et Matussière, dont plusieurs malheureusement, et des plus yualifiés, sont à leur grand regret retenus loin de nous, de pouvoir vous exprimer, en leur nom et au mien, tous nos remereiements pour l'hommage que vous venez de rendre aux trois précurseurs de la Houille blanche.

Ces remerciements, je vous les exprime avec une profonde reconnaissance et une grande émotion.

Dans l'euvre commune réalisée, il ne m'appartient à moins qu's quiconque de discerner et de déterminer les mérites de chacum. Par leur formation, leurs qualités, leur tempérament, Bergìs, Fredet et Mratussière ont marqué leur place dans l'industrie de la Houille blanche.

S'il est vrai que le promoteur du mouvement qui engendra dans notre région la création des premières grandes forces hydraulicues fut incontestablement M. Matussière, il est vrai aussi que les principaux artisans en furent Bergès et Alfred Fredlet qui, presque simultanément, réalisèrent l'installation des hautes chutes de Brignoud et de Lancey. Bergès devait se surpasser lui-même quelques années après, en créant sa chute de 500 mètres, en perçant le lac Crozet, et devait mettre le comble à sa renommée par la vulgarisation de ses magnificues travaux.

La grande mémoire de Fournerron n'est pas diminuée de l'hommage rendu à Fredet, Bergès et Matussière, et la mémoire de l'un quelconque de ces trois derniers ne pent ètre amoindrie du témoignage de reconnaissance accordé aux deux antres.

Vous souvient-il, mon cher Président, qu'il y a quelques semaines nous trouvant réunis autout d'une table amicale, nous parlions des temps hérö̈ques de la Houille blanche. L'un de nous évoquait ses souvenirs d'enfance et se rappelait très nettement avoir vu maintes et maintes fois, Fredet, Bergès et Matussière, qui avaient toujours quelcues travaux en cours d'exécution, venir ensemble aux bureaux des Etablissements Bouchayer et Viallet pour y discuter projets et deris.

Tous trois descendaient du train matinal de Chambéry qui, - à cette époque où il n'était question ni de téléphone ni d'automobile, - amenait à la ville pour leurs affaires, industriels, commerçants et bonnes gens de la Vallée. Alfred Fredet y était monté à Brignoud, Bergès à Lancey, Matussière à Domène. Or, comme vous le savez, ces trois stations, suivant que l'on parcourt la ligne dans un sens ou dans l'autre, se précèdent ou se suivent de près.

N'est-il pas permis de voir un symbole dans ce train vieillot et familier - train de la Houille blanche que nos amis de la Compagnie P.-L.-M. se devraient bien d'électrifier aujourd'hui - déroulant son parcours au pied de Belledonne, emmenant ensemble ces trois pionniers, aujourd'hui à l'honneur, vers leur dur labeur quotidien, vers la réalisation de leur rêve commun, vers l'accomplissement de leurs projets si gros de conséquences pour l'avenir.

Qu'auraient pensé ces trois voyageurs s'ils avaient pu deviner qu'un jour leurs descendants, s'arrêtant à mi-route, à Montfort, seraient transportés par la magie de l'électricité sur ce plateau des Petites-Roches, d'où, entourés de cette élite, qui est une des forces de la France, ils peuvent embrasser, d'un même regard, Brignoud, Lancey et Domène, auréolés d'une gloire commune.

Respectons l'union féconde que cette image évoque, et soyez remercié du fond du cœur, mon cher Président, pour l'éloge magnifique que vous venez de prononcer de ceux dont nous conservons le souvenir avec une piété filiale et une légitime fierté.

Les travaux du troisième Congrìs de la Houllue blanche

Nous avons donné, dans le numéro de mai-juin de notre Revue communication des différents rapports présentés au Congrès. Les travaux se poursuivirent sans interruption jusqu'au 8 juillet, au milieu du concours empressé de nombreux spécialistes.

La section technique confiait à une sous-section le soin d'examiner les propositions visant spécialement l'utilisation de la Houille blanche.

M. Flusin, professeur à la Faculté des Sciences de Grenoble, présidait cette sous-sectión, entouré de MM. Henry Gall, vice-président de la Chambre synctieale des Forces hyclrauliques, et Dupin, secrétaire de la section techniçue.

M. Parodi, ingénieur en chef des services électriques de la Compagnie du Chemin ale fer de Paris à Orléans, traitant de l'utilisation rationnelle, au point de vue technique, de la houlle blanche pour l'électrification des chemins de ler, intéressa vivement l'auditoire. Il examina notamment le cas où les réseaux alimentent uniquement le service du chemin de fer et le cas où les réseaux alimentent à la fois le service de grande traction et des agglomérations importantes à charge industrielle. "En prenant une tension suffisamment élevée, dit-il, on pourra aceepter de transporter l'énergie dans des conditions d'utilisation gui faciliteront beaucoup la conclusion des ententes entre producteurs et consommateurs d'énergie. "Escomptant les progrès de la technique électrique qui permettront, d'ici peu, d'envisager des tensions de l'ordre de 250 a 300 kilovolts, "pour de pareilles tensions, a-t-il conclu, il sera possible de se contenter, pour des transports à 500 kilonìtres, d'une utilisation de 2.000 a 3.000 heures, tout en assurant la rémunération des capjtaux. "

\section{Ce rapport domma lieu à une large diseussion.}

D'autres communications d'un grand intérèt furent présentées par M. Francois Gall, directeur de la Société des Electrodes de Savoie, qui montra que l'électrométallurgiste dispose actuellement de trois types d'électrodes; par M. Jean Gall, ingénieur des mines, qui expliqua comment l'industric du carbure de calcium est particulièrement gènée par les irrégularités de débit de la plupart de nos chutes ; par M. Altmayer, qui traita de l'électrométallurgie par voie humide, et par MM. Mathieu et Sutter, qui exposerent létal des industries blectrothermiques.

M. Johansen, d'Oslo, fit connaitre, à ce propos, l'existence d'un nouveau traitement électrothermique direct des minerais de fer.

Puis le vou suivant a été pris en considération par l'assemblée :

"Que l'aide la plus efficace des pouvoirs publics et des Compagnies de chemins de fer soit apportée aux industries d'alliages exportatrices; que la collaboration la plus intime interviemne entre les divers producteurs d'énergie et les utilisateurs, de manière à réaliser l'emploi intégral et le plus rationnel des puissances disponibles; qu'il soit, en particulier, temu le plus grand compte des besoins considérables d'énergie nécessaire pour la fabrication des alliages destinés à l'exportation, énergie qui pourra éventuellement ètre saisonnière, mais à bas prix. "

La section techuique, placée sous la présidence de M. Rateau, membre de l'Institut, épuisant son ordre du jour, entendit l'exposé de MN. Eydoux et Goua, qui montrèrent que les cheminées d'équilibre, en évitant la propagation des coups de bélier d'ondes donnant des surpressions importantes, sont le complément indispensable des canaux d'amenée en charge.

Le problème de la houille bleue fut exposé par M. de Rouville : la question des essais du matériel des usines hydrauliques, par M. Laurent.

Ayant pris connaissance du rapport de M. Camichel, qui montre tout l'intérèt des expériences exécutées préalablement à la construction sur des modèles réduits, la section a abordé la discussion du rapport de MM. Vennin et Delaly, cui ont insisté sur l'aide que les laboratoires peuvent donner à l'industrie pour la recherche des perfectionnements aux turbines modernes.

M. Leroux a lait connaître à ce propos les premiers résultats obtenus au laboratoire de Beauvert, dans des mesures de haute précision sur le débit des tuyères. La section a pris en considération les conclusions de ces rapports.

Considérant que l'industric a de plus en plus besoin du coefficient de mesures avec une précision qui ne peut ètre atteinte que dans les laboratoires et que cette question intéresse à la fois l'Etat et les collectivités, cette section a émis le vœu "que les laboratoires hydrotechniques soient largement aidés dans leurs recherches par les subventions de l'Etat et par celles des collectivités et des sociétés privées ",

La section a émis ensuite le vou : " que l'Etat organise la mesurc des débits solides des principaux cours d'eau, comme il a déjà organisé celle des clébits liquides et que l'on recherche, par des expériences de laboratoire au besoin, les lois quil régissent l'écoulement des matières solides".

D'intéressantes discussions furent soulevées par l'examen des questions inscrites à l'ordre du jour des sections administratives, économiques et financières. 
M. Raoul Manchard présenta de façon saisissante une vue d'ensemble de la question Houille blanche dans l'économie nationale en France et à l'étranger.

La thèse des partisans de l'énergie termique affronte la thèse des partisans de l'énergie hydraulique à la suite de la communication de M. Mercier, administrateur délégué de l’Union hydroélectrique qui développa

de la Brosse, inspecteur général des Ponts et Chaussées ; Brylinski, délégué général de l'Union internationale des Producteurs et Distributeurs d'énergie électrique; Eydoux, professeur à l'Ecole des Ponts et Chaussées; Augustin Blanchet, président de la Société hydrotechnique de France; Aimé Bouchaver, président de l'Association des Producteurs des Ilpes francaises, etc. des considérations concrétes sur le prix de revient de l'énergie de provenance hydraulique, expliquant que le prix des installations de ce genre n'est pas prohilsilil el préconisant diverses mesures pour leur développement en France. De son côté, M. Arbelot, ingénieur général de la Société des grands travaux de Marscille, formula la crainte que les conditions de la concurrence entre l'énergie de provenance thermique et l'énergie de provenance hydraulicue ne soient très rapidement funestes à celle-ci. D'apres lui, il convient de proléger les installations hydrauliques par l'établissement d'une taxe sur les kilowatts-heures d'origine thermique, taxe affectée à la constilution d'un fonds spécial d'aide à la cons-

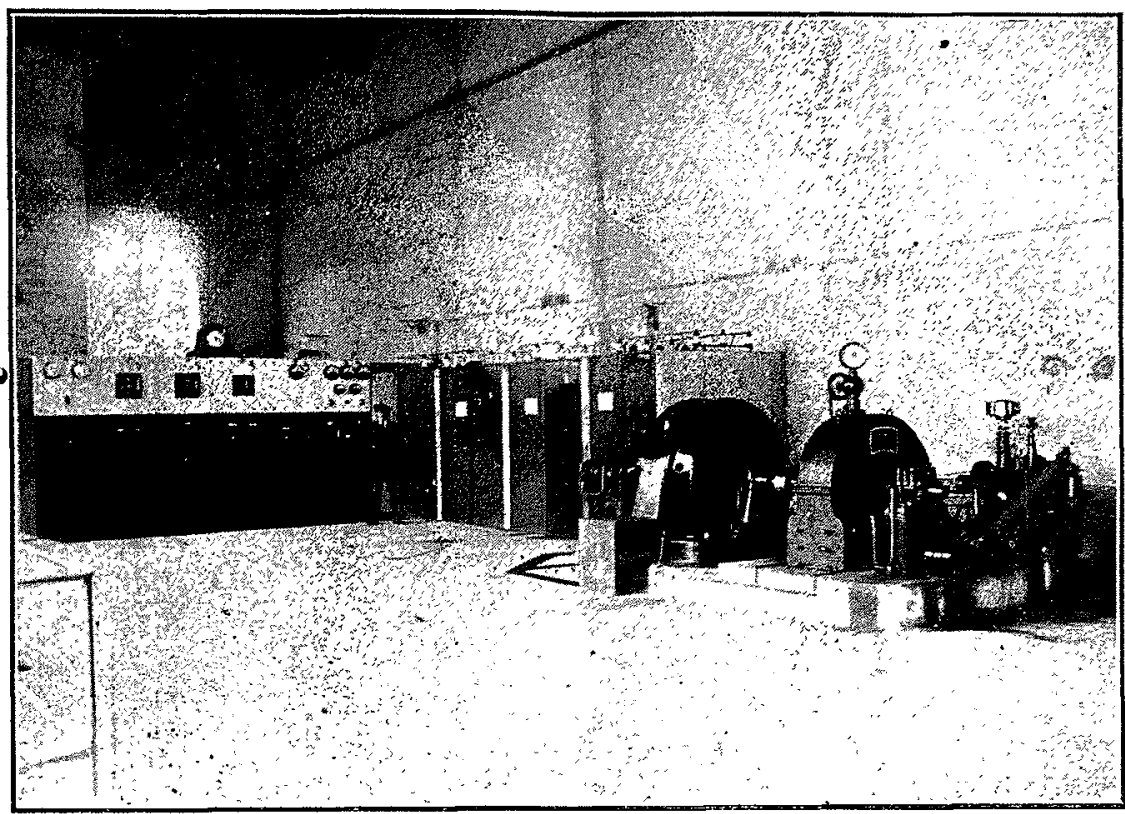

Société Hydro-Electrique de Fure et Morge et de Vizille Centrale semi-automatique de Loulla, de $500 \mathrm{KVA}, 11.000$ volts truction des usines hydrauliques ef par la participation de l'Etat, apportant, par ses avantages, une aide véritable de trésorerie.

MM. Bloch et Maroger ont étudié comment on pourrait faciliter le crédit aux entreprises de la Houlle blanche. Ils ont préconisé l'organisation d'une cooldination ćtroite entre les différents secteurs de production et de distribution, l'intervention financière de l'Etat pour la construction des grands réseaux, l'application d'un index économique à l'énergie hydraulique et divers modes de financement des entreprises nouvelles. Cie dernier point amena une discussion assez animée sur la possibilité d'émettre des actions or, avec intérêts payables en or, procéclé contre lequel s'élève $M$. Bloch et qui ne parut pas rallier les suffrages de l'assemblée.

SÉance comaune du 8 JUILlET ENTRE LE CONGRÈS DU SYNDICAT DES Producteurs et DisTRIBUTEURS D'ÉNERGIE ÉLECTRIQUE ET DU TROISIÈME CONGRÈS DE LA Houille blanche.

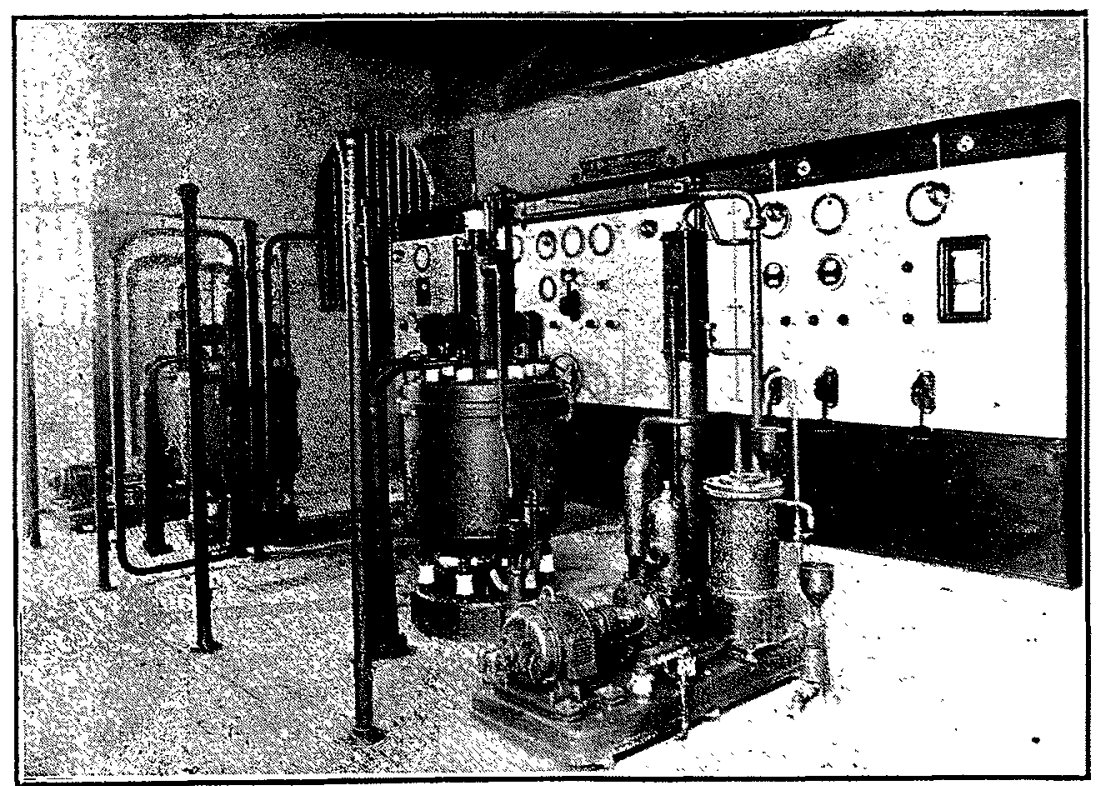

La sous-station de la Galochère

2 groupes redresseurs à vapeur de mercure, type Brown-Boveri, de $285 \mathrm{Kw}$ chacun, 600 volts, cote continu, alimentant les lignes de tramways de la Régie départementale des Voies ferrées du Dauphiné
Un exposé succinct des divers rapports déjà discutés en séance du Congrès de la Houille blanche fut présenté et les vœux suivants ont été adoptés d'un commun accord entre les deux Congrès.

"Que les sociétés ayant pour but la production, le transport et la distribution de l'énergie électrique et disposant d'une puissance de plus de 50.000 kilowatts, se préoccupent d'adopter une organisation de leur rxploitation comportant des services similaires, ce cui faciliterait la comparáison des résultats obtenus par chacun de ces services ;

Que les résultats de l'organisation adoptée par chacune de ces grandes exploitations soient centralisés pour permettre cette comparaison et en déduire la nature des améliorations à adopter ;

"Que l'organe centralisateur choisi soit également renseigné sur la question des liaisons téléphoniques, afin de mettre le plus vite possible en évidence les meilleurs procédés à cmployer au point de vue technicue, administratif et financier."

BANOUET DL 8 JUILLET aU Granase MUNicipal de Grenoble, RÉnNisSANT LES DEUX CONGRÈS.

Le 8 juillet, à 12 h. 30 , un banquet, organisé par les deux Congrès réunissait, sous la présidence de M. Gabriel Cordier, président d'homeur de la Chambre syndicale des Forces hydrauliques, près de cinq cents convives. A l'heure des toasts, M. Marlio, président du Congrès de la Houille blanche, montra la profonde communauté d'intérèts existant entre les deux groupements auxquels revenait le mérite de l'organisation de ce banquet « et, a-t-il ajouté, si la collaboration féconde pent s'instituer entre eux,

Une séance commune a réuni, dans la matinée du $\&$ juillet, à l'amphithéâtre Marcel Reymond, les deux Congrès en vue d'arriver, sur les questions inscrites à l'ordre du jour du Congrès de la Hoville blanche intéressant spécialement les producteurs et distributeurs d'énergie électrique, à l'expression de vœux communs.

M. Marlio, président de la Chambre syndicale des Forces hydrauliques, présidait cette séancc, entouré de MM. Cahen, président du Syndicat des Producteurs et Distributeurs d'énergie électriçue; elle est grandement facilitée par M. Cahen, dont on comnait la compétence et le caractère ".

Au nom du Congrès des Producteurs et Distributeurs d'énerigic électrique, M. Cahen exprima l'opinion que l'opposition qui paraissait irréductible entre la houille blanche et la houille noire était vaincue : "Appuyés l'un sur l'autre, jonctionnés rationnellement, mulitplient, par leur union, leurs possibilités réciproques de réalices deux grands modes de production hydraulique et thermique 
sation. "Examinant ensuite les besoins croissants de la collectivité auxquels lindustrie doit faire face: "Nous savous tous, dit-il, que la limite d'activite de notre industrie est loin ditre alteinte et nous croyons que, dans un avenir rapproché, les besoms en énergie atteindront des proportions dont on n'a pu se fiare aueune idie jusquà présent. L'électrification des campagnes en liance sera réalisée par étapes plus ou moins rapides. Il semble donc que. mème avec les estimations les plus modérées, on pent prévoir, pour toute la France, le doublement de la consommation actuelle dans une période de dix ans. Il faut done que les affaires de distribution doublent aussi, pendant cette période, leurs disponibilités en énergie et leurs moyens de transport et de distribution. D'où la nécessité de nouveaux capitaux considérables que l'on peut évaluer à près d'un milliard de franes par an pour cette période de dix ans. Comment réussir si on ne permet pas ou si on compromet la prospérité des affaires qui sont obligées d'y avoir recours?"

En un discours plein d'érudition, M. Lépine, président de la Chambre de commerce, fit ressortir les raisons qui désignaient Grenoble pour qu'y soit temu ce Congrès. "La Chambre de commerce ne pouvait, dit-il, oublier que Grenoble a été le véritable berceau de la houille blanche; car, si, en 1827, c'est ì Pont-surl'Ognin que Fourneyron inventa et installa la première turbine, c'est dans les environs immédiats de Grenoble, à Lancey, à Brignoud et Domène que, en 1868, Bergès, Fredet et Matussière utilisèrent industriellement, pour la fabrication du papier, les premières turbines fonctionnant sous de hautes chutes; c'est entre Vizille et les halles de Grenoble que, en 1883, Marcel Desprez fit ses mémorables expériences de transport de l'énergie à distance au moyen de l'électricité.

Puis M. Lépine fit une savante description des grands travaux de la région grenobloise qui lui assurent le record des hautes chutes.

D'autres allocutions furent prononcées par $M$. Fantoli Gaudenzio, déléguć d'Italie, au nom des délégations étrangères ; par M. Mistral, maire de Grenoble ; par le préfet de 1 'Isère et par $\mathrm{M}$. Théodore Tissier, président de la section des travaux publics au Conseil d'Etat. Enfin, M. Cordier, en un discours très applaudi, clégagea l'enseignement à tirer de la manifestation qui va prendre lin : "Que de cbemin parcouru, a-t-il dit, quand on se reporte à ce Congrès de la Houlle blanche de 1902, qui fut une révélation et en souvenir duquel je vous demande d'arlresser l'hommage de notre reconnaissance à la mémoire d'un homme qui fut un grand serviteur de notre cause, j'ai nommé Charles Pinat, fonclateur et premier président de la Chambre syndicale des Forces hydrauliques."

Après avoir examiné les différentes inesures à prendre pour faciliter la mise en cuvre des chutes, M. Cordier déclara que l'autorité des vœux émis par le Congrès hâterait certainement l'obtention de décisions importantes.

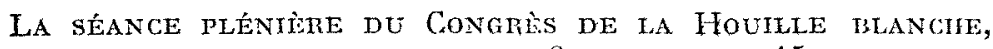
ADOPTION DES VEUX, CLOTURE 8 JUILLET, A 15 HEURES, A L'Ampinthétere Marcel-Reymond.

La séance plénière a eu lieu à l'amphithéâtre Marcel-Reymond, immédiatement après le banquet du Gymnase municipal.

Au cours de cette séance furent adoptés de nombreux voux,

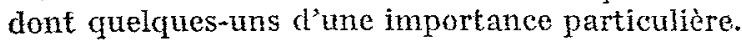

M. Louis Marlio, président du Congrès, fit en quelques mots un lumineux exposé des résultats du Congrès. Dans l'ordre technique, le Congrès a permis de mesurer les progrès sclentifiques réalisés dans la construction hydraulique depuis vingt-trois ans. Dans
Yordre administratif, il lait ressortir le désir d'appliquer la loi dans un sens plus pratique et avee plus de souplesse. Dans l'ordre ćconomique, sans nier la crise, le Congrès a cherché à la délimiter et à la réduire en proposant des mesures propres à diminuer le prix du kilowatt ou à lui assurer une utilisation plus rationnelle. "Aussi, conclut M. Marlio, nous eroyons que la crise n'est que lemporaire et que la confiance renaitra. C'est, dil-il encore, paree que nous apportons les resultats de nos efforts, que nous nous trouvons fondés à demander aide a l'Etat. "

Les voux émis par la section technique ont été cléfinitivement adoptés.

Sur la proposition de cotte section, le Congres, consiclerant que le développement des installations hydro-électriques et l'augmentation de la puissance des usines génératrices donnent une importance croissante à la question des essais, a, cle plus, émis le vou :

$1^{\circ}$ Que les exploitations prévoient dans leurs marchés ou cahiers des charges des essais systématiques et complets à effectuer au monent de la mise en route de leurs installations;

$2^{\circ}$ Que les conditions de ces essais soient nettement précisces dans ces marchés aux cahiers des charges et soient, clans un but d'unification et de comparaison, conformes aux instructions et au Code d'essais de la Société hydro-technique de Franeo ct, autant que possible, conficies a cet organisme officiel et indépendant ;

$3^{\circ}$ Quo les exploitants prevoient, des la construction de leur ouvrage, les dispositif's de mesure nécessaires aux essais;

$4^{\circ}$ Que des mesures de rendement soient faites périodiquement afin de maintenir, par ce controle permanent, le rendement clu matériel à la valeur maximum compatible avec la considération des frais d'entretien ;

$5^{\circ}$ Que les résultats des essais présentant un caractère technique et non commereial soient publiés par la Société hydrotechnique de France, afin de fournir aux constructeurs et aux exploitants tous les eléments qui peuvent leur itre utiles pour le perfectionnement de la technique hydro-électrique.

VaUX EMIS DANS L'ONDRE ADMINISTRATIF, ECONOMIQUE ET FINAXCIER.

En dehors des voux techniques, le Congrès de la Houlle blanche a adopté des veux ayant un caractère administratif, économique et financier, dont voici les principaux :

Au sujet des règles de droit international concernant l'utilisation de la force motrice des cours d'eau ou des lacs appartenant à deux pays ou limitrophes entre deux pays, le Congrès émet le vou:

$1^{\circ}$ Que l'Etat français provorue des maintenant une étude, concertéc avec les Etats voisins, des aménagements de forces hydrauliques susceptibles d'intéresser ces Etats, afin d'éviter toute perte de temps le jour où ces aménagements deviendront urgents ;

$2^{\circ}$ Qu'il réponde avec empressement aux demandes analogues que lui présenteront les Etats voisins et s'efforce d'arriver à des solutions également satisfaisantes pour tous les intérêts en présence;

Au sujet d'une réglementation de l'importation d'énergic électrique :

Que la conclusion de tout nouveau contrat d'importation soit soumise a l'approbation du ministre des Travaux publics et que 
cette approbation soit donnée sur avis d'une commission comprenant quatre représentants de l'administration, deux représentants de la Chambre syndicale des Forces hydrauliques et deux représentants du Syndicat professionnel de producteurs et distributeurs d'clectricité.

D'autres voux concernent : $1^{0}$ les améliorations à apporter aux règlements qui régissent les relations entre l'Ftat et les sociètès ou particuliers, qui projettent d'écuiper ou qui exploitent une chufe d'eau ou une ligine de transport à haute tension avec des fiers propriétalres de terrains possesseurs de droits d'eau ou les précídents usagers : $2^{\circ}$ les améliorations des conditions de vente ou de transport de l'énergie imposées aux concessionnaires de chutes d'eau ou de réscaux à haute tension au profit du public.

Dans l'ordre économique et financier, lè Congrès a réclamé uné politique de dégrèvements fiscanx temporaires, analogue à cclle suivie en matiere de construction d'immeubles, en vue d'abaisser le prix de revient des installations hyclro-cilectriques et d'améliorer les condilions de leur fonctionnement. Il a émis le vou notamment: "Qu'il soit constitué par la Chambre syndicale des Forces hydrauliques un organisme collectif privé, dont les services seraient mis a la disposition des industriels pour assurer le meilleur rendement he leurs installations hydro-électriques, par une surveillance méthodique et continue analogue à celle qui est exercée par les diverses associations de propriétaires d'appareils à vapeur. "

Enfin, pour remédier à la situation précaire crééc aux entreprises d'énergic hydraulique par les conditions économiques et financières actuelles, le Congrès a adopté un vou dont l'importance n'échappera pas et qui aura sürement, un certain retentissement :

Considérant, a-t-il été déclaré, que, pour remédier à cette situation, il est nécessaire que ces entreprises soient mises à mìme d'offrir au public des titres dont le revenu varie avec le pouvoir d'achat réel de la monnaie;

Que, pour le moment, les sociétés ne peuvent que créer des titres à jevenu variable, comportant, par exemple, un taux d'intérèt fixe et une rémunération supplémentaire, fonction de l'index économique;

\section{Le Congrès émet le voeu :}

$1^{\circ}$ Que les sociétés concessionnaires de chutes d'eau ou de lignes de transport soient autorisées à émettre des obligations bénéficiant d'une garantie de change;

$2^{\circ}$ Que les tarifs maxima applicables à la vente de l'énergie hydraulique comportent un terme correctif, fonction de l'index économicque, le coefficient de ce terme correctif étant calculé notamment, dans chaque cas d'espèce, d'après la constitution du capital de premier établissement.

\section{Vou COMMUN AUX DEUx CONGRìs}

Au sujet des charges fiscales des usines hydro-électriques, le vou "que l'Etat veuille bien accorder temporairement des exemptions d'impốts et àutres avantages fiscaux, pendant une période d'au moins quinze aus à compter de leur achèvement aux usines hydroélectriques et aux lignes de transport a haute tension, en vue de faciliter l'aménagement des richesses hydrauliques du pays ". fut adopté par les deux Congrès de la Houille blenche et des producteurs et distributeurs d'énergie élcetricue.

M. Marlio remercia ensuite tous ceux cui avaient apporté leur collabotation au Congrès. Il ajouta que le Congrès avait cherché ì stabiliser et à réduire la crise économique actuelle, crise temporaire gui ne devait pas cntaner la confiance de la France.

M. Marlio dit aussi qu'il fallait demander au Gouvernement un allègement des impôts sur l'établissement des chutes d'eau et des transports a haute tension, li création de chutes nouvelles apportant de noivelles ressources a l'Etat.

Il termina par une déclaration très nette en faveur de la liberté, c'est-à-dire en faveur du régime de la concession en affirmant la répugnatice du Congrès pour le régine de la régie par l'Etat. La séance se terminait vers 17 heures et une conférence de M. Klohinger, ingénieur de la Soclété Brown-Boveri avait lieu itmmétliatenent après, pentant que queltues congressistes, sous la direction de M. Aimé Bouchayer, rendalent visite au Comité des Etutiants etirangers.

\author{
CONFERTNCE DE M. KLONINGER

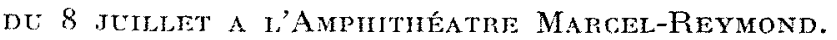

Immédiatement après la clòture du $3^{\mathrm{e}}$ Congrès de la Houille blanche avait lieu, dans le même local, à l'amphithéâtre MarcelRaymond, en l'honneur des deur Congrès et sous les auspices de l'Association "la Houille blanche "des Anciens Elèves de l'Institut Electro-technique de Grenoble, la conférence de M. Kloninger, ingénieur de la Socićté Brown-Boveri. délégué par la Compagnie Electro-Mécanicue. Autour du conférencier prenaient place M. Barbillion, directeur de l'Institut polytechnicue de Grenoble, M. Msirlin, président du Groupe dauphinris de l'Association La Houlle blanche, M. V. Sylvestre, vice-président de l'Association.

Le conférencier fut présenté en termes particulièrement élogieux par M. Barbillion, gui remercia M. Sylvestre d'avoir bien voulu organiser cette conférence.

Devant une assistance nombreuse constituée en grande partie par les tenanciers des Congrès de la Houille blanche et du Syndicat des producteurs et distributeurs d'énergie électrirque, les directeurs des grandes Sociétés régionales de distribution, des professeurs de l'Institut polytechnique et les anciens élèves de l'Institut, M. Kloninger exposa avec une maîtrise incomparable les grands problèmes de transport d'énergie à tension constante, ainsi que les applications des régulateurs automatiques, en particulier les régulateurs à action rapide.

Il a montré à quel degré l'introduction de l'automatisme dans les stations centrales pouvait introduire des économies d'exploitation et mème, en dépit de l'appiarence paradoxale de cette affirmation, de sécurité dans la marche des usines.

Il a fait remarquer combien la région des Alpes françaises était en avance sur les autres régions de la France pour les applications des appareils de réglage automatique. Fn effet, la plupart des grandes centrales hydro-électriques de nos Alpes possèdent des régulateurs réglant automatiquement non seulement la tension des génératrices, mais aussi la répartition de l'énergie réactive entre les génératrices des centrales fonctionnant en parallèle. Les prohlèmes les plus divers $y$ sont résolus, tels que : réglage à pulssance constante des moteurs commandant des défibreurs, à la Société des Papeteries de France ì Mancey; chez Dodo, à Domène, aux Papeteires Peyron, à Vizille; réglage à vitesse constante des machines à papier, aux papeteries Navarre, à Champ-sur-le-Drac, à la Société des cartonneries de la Rochette; réglage à puissance constante des fours électriques à la Société d'électro-chimie d'électrométallurgie d'Ugines, sur des fours à acier à 4 électrodes de 20 tonnes: réglage à cos coustant des compensateurs synchrones à l'usine Enferton à Rives, à la Biscuiterie Brun à Grenoble. On y trouve également des dispositifs de mise en parallèle automatique :

a) d'alternateurs entr'eux à la centrale d'Avrieux, près Modane ;

b) d'une centrale thermique et d'une centrale hydraulique, aux Cartonneries de la Rochette (Savoie);

c) de deux centrales hydrauliques aux Papeteries Peyron, à Vizille:

d) de 3 réseaux 26.000 volts dans le grand poste des Ftablissements Bouchayer et Viallet, ì Grenoble.

Enfin la centrale de Loubla, qui utilise une chute de 360 mòtres, déversoir des lacs de Laffrey, a été équipée en 1923 par la Société Hydro-blectrique de Fure et Morge et de Vizille en dispositif semiautomatique.

Une importante discussion technique suivit la conférence au cours de laquelle M. fillio, directeur de la Société Hydro-électrique de Fure et Morge et de $\mathrm{V}^{\mathrm{r}} \mathrm{z}$ ille exposa avec schéma et croquis à l'appui les raisons pour lesquelles sa Société avait été conduite à anvisager l'équipement automatique de la station génératrice de Loubla.

\section{LES VISITES D'USINES.}

Les dirfórents Congrès mirent à profit les quelques moments de loisir qu ils pouvaient avoir entre les séances poutr visiter les usines intéressantes de la région, c'est ainsi cu'ils visitèrent la sousstation dé la Galochere de la Société Hydro-électrique de Fure et Morge et de Virille, l'usine du Baton des Etablissements KellerI.eleux, les usines d'Ugine de la Société d'Electrochimie, l'usine du Drac-Romanche, le laboratoire de Beauvert de la Société Hydrótechnicue de France, la position du batrage du Chambon, ete: 
Visile de la sous-station de la Galochire, alimentant les tramways de la Régie départementale des voies ferrétes du Dauphiné.

Cette visite faisait partie du programme du Congris de la Société française des Electriciens, elle cut lieu le 10 juillet aprèsmidi.

La sous-station de la Galochère alinente l'important ríseau de tramways Grenoble, Lriage, Vizille. Lancey, Froges, de la Régie départementale des voies ferrées du Dauphine.

De nombreux congressistes de la Société Française des Flectriciens et du Syndicat des producteurs et distributeurs d'énergie clectricue furent transportés à la sous-station de la Galochère à l'aide de tramwars spéciaux qui avaient été mis à leur disposition par M. Codron, directeur de la Régie départementale des voies ferrées du Dauphint.

Les visiteurs furent recus à la Galochère par M. Gillio, directeur de la Société Hydro-électrique de Fure et Morge et de Vizille, M. Braisaz, ingénieur à cette Société et M. V. Sylvestre, ingénicur

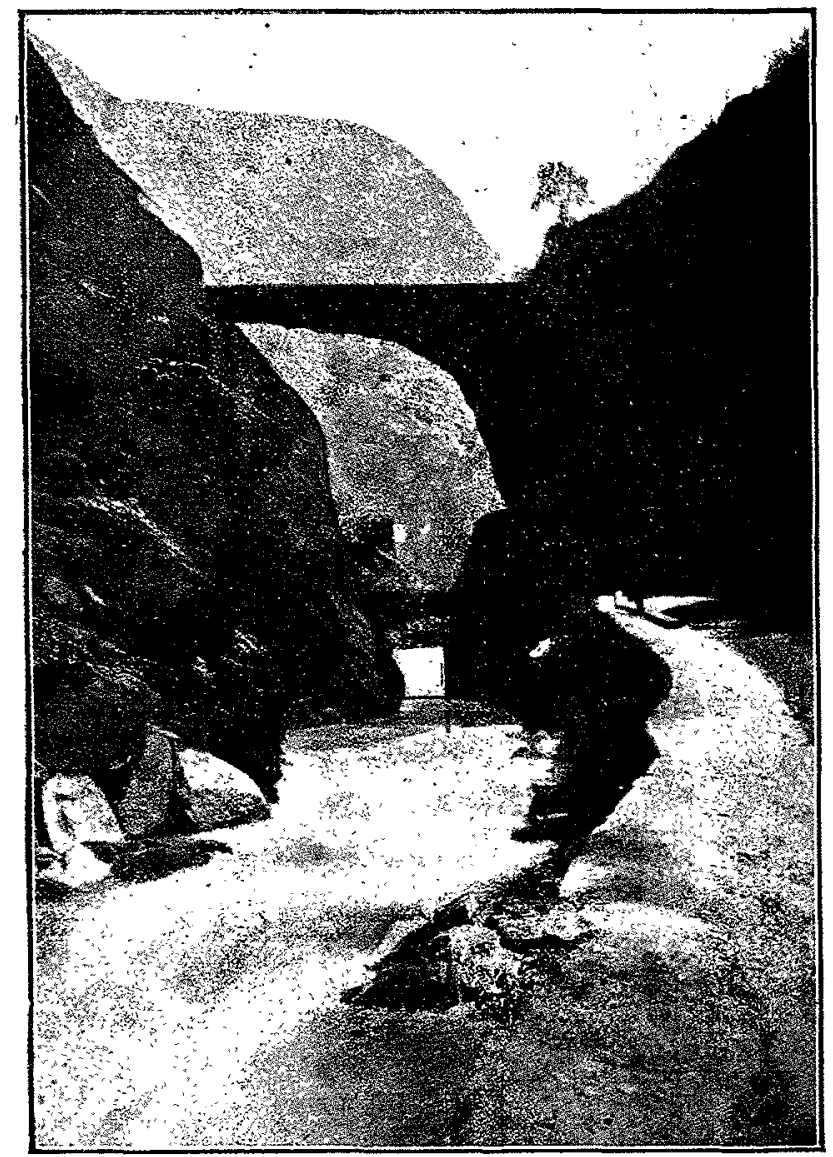

Emplacement du Barrage-Réservoir de Chambon - Vue de l'aval L'horizoniale supérieure esi au niveau du conronnement dubarrage (Projet II. Fredel)

à la Compagnie Electro-mécanique, vice président de la Houille blanche.

M. Gillio leur souhaita la bienvenue, les remercia d'être venus si nombreux malgré le programme si chargé des différents Congrès, puis rappela qu'en 1902, lors du premier Congrès de la Houille blanche qui eut lieu à Grenoble, la plupart des congressistes visitèrent la sous-station de la Galochère. Cette sous-station était alors équipée à l'aide de trois commutatrices "Westinghouse " de chacune $170 \mathrm{Kw}, 600$ volts 750 tours. qui étaient les premjères machines du genre à 50 périodes installées en France. Ces commutatrices, qui fonctionnaient depuis le 15 aloût 1902, avec entière satisfaction, arrivaient toutefois à un état d'usure faisant craindre pour la sécurité de l'exploitation; d'un autre côté, le rendement d'ensemble n'était plus compatible avec une installation moderne.

C'est alors que la Société Hydro-électrique de Fure et Morge et de Vizille, devant l'extension prise par la Régie départementale des voies ferrées du Dauphiné, remplaça il y a quelques mois les commutatrices par des machines à grand rendement à touest charges, dont le fonctionnement soit le plus simple possible : des redresseurs à vapeur de mercure à grand debil lype "Brown-Boveri".

M. Gillio fit la description de la nouvelle installation, composée de deux eylindres redresseurs de $285 \mathrm{Kw}$. chacun, 475 ampères, 600 volts côté continu, le courant d'alimentation se faisant à l'aide du réseau général triphasé 10.000 volts de la Société de Fure et Morge, puis fit opérer un certain nombre d'arrêts et de mises en marche de la sous-station.

Il exposa ensuite, d'une façon tout a fait remarquable, les qualités techniques des redresseurs à vapeur de marcure, grand rendsment à toute charge, facilité de manouvre, simplicité, etc.

M. Sylvestre domna aux visiteurs des renseignements détaillés sur la composition des cylindres redresseurs, et fit ressortir les différences essentielles qu'il y avait entre ces nouvelles machines et les groupes tournants.

Nous avons remarqué parmi l'assistance:

MM. Lebaupin, „président de la Société Française des Electriciens; Dumont, administrateur de la Compagnic Générale des

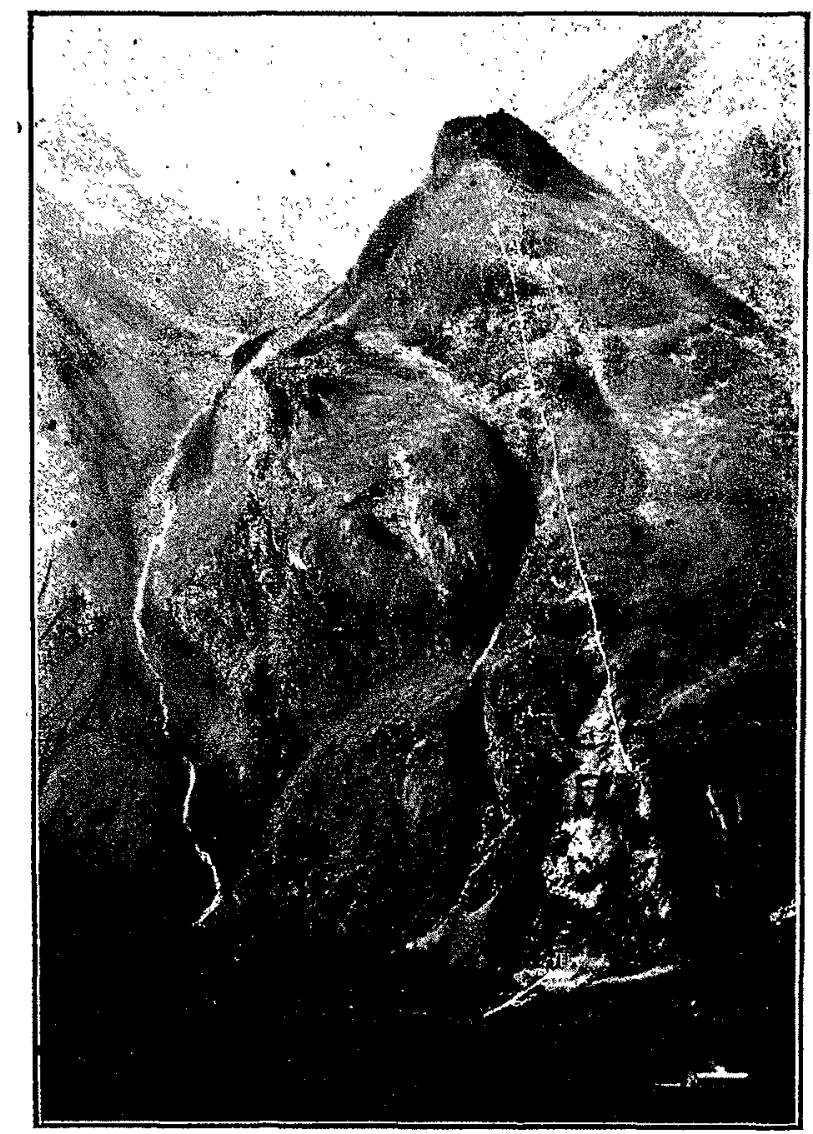

Vue générale de la chute du Bâton, la plus haute chute de France (1.045 mètres)

(On aperçoil en bas l'usine qui est aménagée dans le rocher)

câbles; Desombre, administrateur-délégué de la Compagnie Electro-mécanique; Bouyond, ingénieur-conseil ; Béthenod, ingénicurconseil; Geymond, professeur; Joucla, directeur de l'agence de Lyon de la Compagnie Electro-mécanique; Maure, délégué de la Gazetle des Alpes; Maréchal, de la Compagnie du Gaz de Lyon, etc., de nombreux ingénieurs des régions de Paris, Lyon, St-Etienne et Marseille.

A l'issue de la visite, un vin d'honneur fut offert au splendide Parc des Anguines, par la Société Hydro-électrique de Fure et Morge et de Vizille.

M. Gillio excusa tout d'abord M. Lépine, président de la Chambre de Commerce et administrateur-délégué de la Société Ilydro-élcctrique de Fure et Morge et de Vizille, empêché par des devoirs impérieux à la Chambre de Commerce, puis leva son verre en l'honneur de ses hôtes éminents, en particulier de M. Lebaupin, président de la Société Française des électriciens et félicita M. Desombre, administrateur-délégué de la Compagnie Electro-méca- 
nique du tour de force qui avait été réalisé par sa Société, de meltre sur pied, en trois mois, une sous-station qui constitue une nouveauté clans la région du Sud-Est et apporte une amélioration considérable dans la technique des sous-stations d'alimentation en courant continu, des tramways départementaux.

Enfin M. Lcbaupin remercia M. Gillio et M. Desombre, fit un rapide exposé des avantages des redresseurs à vapeur de mercure, signala qu'ils constituaient des machines précieuses pour l'électrification des chemins de fer français, leva son verre à la santé de M. Lépine, à celle des congressistes et à la réussite du Congrès de la Sociélé Franģaise des électriciens.

\section{La plus haute chute de France, la cirute de Baton,} 1.0.15 METPLS (1).

Juscu'à ces (lerniers mois (mars 1925), la plus haute chute des Alpes et aussi de France était celle dle Fond-cle-France, qui utilise les lacs des Sept-Laux (1.032 mètres).

Cette hauteur vient d'être légèrement dépassée par la chute excessivement intéressante que vient d'équiper M. Charles-Albert Keller, l'électrométallurgiste de Livet. Sous l'action de M. Keller, les usines de Livet ont jouć un rôle de premier ordre dans l'histoire de l'électrométalluirgie. On lui doit cle précieuses découvertes, particulièrement en ce qui concerne la fabrication de la fonte synthétique, de l'acier au four électrique, Ja refusion électrique du ferro-manganèse pour son introduction dans l'acier. Avant la guerre, les usines de livet produisaient environ 16.000 tommes de carbure de calcium, 7.000 tomes de ferro-alliages et quelques tonnes de fontes synthétiques. M. Keller a considérablement développé pendant la guerre la fabrication des fontes spéciales synthétiques. Il a créé une fonclerie de projectiles en fonte aciérée, calibres $220-240$ et 100 , fabriqués avec la fonte synthétique. Il a aussi réalisé dans ses usines de Livet, à l'aide de la Houille blanche, tout un cycle métallurgique nouveau partant de tournures d'acier pour arriver au projectile fini. Pour pouvoir réaliser un tel programme, il fallait aux usines de Livet de plus en plus de force, et la centrale de Livet, bien qu'elle ait une puissance de 10.000 kilowatts, ne pouvant plus suffire, M. Keller a d'abord équipé en pleine guerre la belle centrale des Vernes $(1.000 \mathrm{KW})$, puis a jeté ses vues sur le ruisseau du Bâton.

Le ruisseau du Bâton prend sa source sur les pentes est du massif de Belledonne, à plus de 2.500 metres, se précipite dans la vallée de Livet par la cascade de Baton, et se jette dans la Romanche, à environ 3 kilomètres en amont du village de Livet. Le ruisseau du Bâton est dérivé, à l'altitude de 1.760 mètres, dans un tunnel creusé à grande profondeur dans la roche ; une chambre de décantation est en souterrain dans le rocher, avec évacuation par une galerie-fenêtre. Le tunnel a environ 1.103 mètres de longueur, de 6 à 7 mètres carrés de section, pouvant constituer une réserve de 7.800 mètres cubes. Le tunnel aboutit à une chambre d'eau située à 30 mètres de profondeur dans le rocher ; cette chambre est munie du reniflard de la conduite et d'une vanne automatique; celle-ci, en cas de rupture de la conduite, se ferme automatiquement sous l'action de la survitesse de l'eau; elle peut aussi se mancuvrer depuis l'usine à l'aide d'un câble souterrain. Une conduite forcée

(1) Voir l'ouvrage a La Houille Blanche», par V. Sylvestre, chez Rey, éditeur, Grande Rue, Grenoble. de 1.565 mètres de longueur part de la côte 1.753 pour aboutir à. l'usine située à la cote 715 . La conduite a 540 millimètres de diamètre et est en tôle d'acier rivé entre les cotes 1.753 et 1.249 , et en tôle d'acier soudé entre la cote 1.249 et l'usine. Afin d'éviter le gel, et pour la protéger contre la chute des rochers, la conduite est enterrée à 2 mètres de profondeur. Toutefois, à partir de la cote 859 , la montagne était tellement à pic que l'on a été amené à percer une véritable cheminée inclinée à $61^{\circ}$ recevant les 200 derniers mètres de la conduite. Le montage de cette conduite a été extrêmement délicat et n’a pu être réalisé que grâce à la ténacité de M. Keller et l'expérience consommée des constructeurs grenoblois. L'usine génératrice est excessivement curieuse; il n'y a pas de bâtiment à proprement parler, ou plutôt le bâtiment est la montagne elle-mème; l'usine est du type "troglodyte ", comme celle du Bas-Laval, c'est-à-dire qu'elle est complètement taillée dans le rocher. Cette disposition était la seule rationnelle, étant données les chutes de pierres importantes qui se produisent à Bâton. La conduite débouche à l'arrière de la grotte créée par l'usine et aboutit à un collecteur situé dans une galerie parallèle à la salle des machines; les robinets-vannes sont également dans cette galerie, et seules les tubulures allant aux turbines passent dans la salle des machines.

Une galerie d'évacuation a été aménagée entre la galerie du collecteur et l'extérieur, de façon qu'en cas de rupture de conduite dans la galerie ou du collecteur, ou encore des robinets-vannes, il n'y ait aucune catastrophe à redouter. Toute l'installation électrique, tableaux, poste de transformation, est faite dans le rocher; toutes les lignes intérieures passent en caniveaux montés sur le rocher. Le canal de fuite est à double branche, taillé dans le rocher; il aboutit à une chambre de débit et gagne la Romanche par une conduite en ciment armé. L'usine est équipée par deux groupes turbine-alternateur de $3.300 \mathrm{cv}$. chacun; la tension de 4.350 volts des alternateurs est élevée à 26.000 volts, tension du réseau de la Société Fure et Morge. Une ligne partanı́ du Bãton va rejoindre l'usine de Jouchy, pour être connectée au réseau général qui alimente la ville de Grenoble. L'énergie électrique totale de l'usine du Bâton peut être évaluée à environ 25 millions de lîlowatts-heure. L'usine du Bàton sera utilisée au moment des basses eaux pour alimenter, sur un nombre d'heures réduit, le réseau de la ville de Grenoble, avec réserve d'eau dans le tumnel supérieur, et au moment des hautes eaux, l'énergie électrique sera utilisée à l'alimentation des fours électriques de l'usine de Livet, et aussi en parallèle avec l'énergie des hautes eaux de l'usine des Vernes, pour l'alimentation d'une station de pompage, dont nous avons déjà parlé, qui sera située près de l'ne Falcon, dans la vallée de la Romanche, au pied du lac Mort (lacs de Laffrey). Cette station de pompage de 4.800 chevaux de groupes moteur-pompe réversibles, permettra de remplir la réserve de 4.500 .000 mètres cubes du lac Mort, surélevé de $2 \mathrm{~m}$. 30, en vue de l'utilisation de cette réserve pendant les mois d'étiage, de la Romanche et du Bâton.

M. Keller résoudra donc, avec son bassin d'accumulation du lac Mort, le problème de faire fonctionner, à puissance sensiblement constante, l'ensemble de son domaine hydro-électrique constitué par ses usines de Livet, des Vernes et du Bâton ; les deux premières seront elles-mêmes régularisées à l'étiage d'hiver, à l aide de l'accumulation du barrage du Chambon.

Il s'agit là de problèmes excessivement intéressants, et d'une usine des plus curieuses, qui a fait l'objet de nombreuses visites de la part des techniciens qui ont suivi les différents congrès de Grenoble. 